\title{
Correction \\ Correction: Wang et al. Parametric Formula for Stress Concentration Factor of Fillet Weld Joints with Spline Bead Profile. Materials 2020, 13, 4639
}

\author{
Yixun Wang ${ }^{1}$, Yuxiao Luo ${ }^{2}$ and Seiichiro Tsutsumi ${ }^{1, *(D)}$ \\ 1 Joining and Welding Research Institute, Osaka University, Osaka 567-0047, Japan; \\ wang.yixun@jwri.osaka-u.ac.jp \\ 2 Department of Structural Engineering, Tongji University, Shanghai 200092, China; 1410210@tongji.edu.cn \\ * Correspondence: tsutsumi@jwri.osaka-u.ac.jp; Tel.: +81-6-6879-4887
}

check for updates

Citation: Wang, Y.; Luo, Y.; Tsutsumi, S. Correction: Wang et al. Parametric Formula for Stress Concentration Factor of Fillet Weld Joints with Spline Bead Profile. Materials 2020, 13, 4639. Materials 2021, 14, 2433. https://doi.org/10.3390/ ma14092433

Received: 30 March 2021 Accepted: 28 April 2021 Published: 7 May 2021

Publisher's Note: MDPI stays neutral with regard to jurisdictional claims in published maps and institutional affiliations.

Copyright: (c) 2021 by the authors. Licensee MDPI, Basel, Switzerland. This article is an open access article distributed under the terms and conditions of the Creative Commons Attribution (CC BY) license (https:/ / creativecommons.org/licenses/by/ $4.0 /)$.
The authors wish to revise the following from pages 16-18 in the text of Appendix $B$ [1] due to the presence of incorrect information.

The correct text is shown below:

\section{Appendix B}

Formulas for calculating SCFs for T-shape welded joint and cruciform welded joint under tensile and bending stress.

(1) T-shape welded joint under tensile stress:

$$
K_{t}=1+1.39418 \cdot f\left(r_{1} / t\right) \cdot f\left(\theta_{1}\right) \cdot f\left(\left(r_{1} / t\right) \cdot\left(\theta_{1}\right)\right)
$$

where:

$$
\begin{aligned}
& f\left(r_{1} / t\right)=1.824174 \cdot\left(r_{1} / t\right)^{-0.145045}+6.400210 \cdot\left(r_{1} / t\right)^{3}-6.802011 \cdot\left(r_{1} / t\right)^{2} \\
& +3.277059 \cdot\left(r_{1} / t\right)-2.692331 \\
& f\left(\theta_{1}\right)=-2.778232 \times 10^{-1} \cdot\left(\pi-\theta_{1}\right)^{3}+1.531999 \cdot\left(\pi-\theta_{1}\right)^{2} \\
& -3.394907 \cdot\left(\pi-\theta_{1}\right)+4.967130 \\
& f\left(\left(r_{1} / t\right) \cdot\left(\theta_{1}\right)\right)=-1.001612 \times 10^{1} \cdot\left(\left(r_{1} / t\right) \cdot\left(\pi-\theta_{1}\right)\right)^{3}+1.279269 \times 10^{1} \\
& \cdot\left(\left(r_{1} / t\right) \cdot\left(\pi-\theta_{1}\right)\right)^{2}+5.761117 \cdot\left(\left(r_{1} / t\right) \cdot\left(\pi-\theta_{1}\right)\right)+1.181649
\end{aligned}
$$

(2) T-shape welded joint under bending stress:

$$
\begin{gathered}
K_{t}=1-1.129553 \cdot f(T / t) \cdot f\left(r_{1} / t\right) \cdot f\left(\theta_{1}\right) \cdot f\left(\left(r_{1} / t\right) \cdot\left(\theta_{1}\right)\right) \cdot f\left(L_{1} / t\right) \cdot f\left(L_{2} / t\right) \\
\cdot f\left(\left(L_{1} / t\right) \cdot\left(L_{2} / t\right)\right) \cdot f(H / t)
\end{gathered}
$$

where:

$$
\begin{aligned}
& f(T / t)=-9.622916 \times 10^{-3} \cdot(T / t)^{3}-3.157009 \times 10^{-2} \cdot(T / t)^{2} \\
& +1.848086 \times 10^{-1} \cdot(T / t)+2.433139 \\
& f\left(r_{1} / t\right)=6.477191 \times 10^{-1} \cdot\left(r_{1} / t\right)^{-0.258620}+2.860640 \cdot\left(r_{1} / t\right)^{3} \\
& -3.689862 \cdot\left(r_{1} / t\right)^{2}+2.089786 \cdot\left(r_{1} / t\right)-1.238719 \\
& f\left(\theta_{1}\right)=3.450624 \times 10^{-3} \cdot\left(\pi-\theta_{1}\right)^{3}-3.531382 \times 10^{-2} \cdot\left(\pi-\theta_{1}\right)^{2} \\
& +6.008736 \times 10^{-2} \cdot\left(\pi-\theta_{1}\right)+8.576512 \times 10^{-2} \\
& f\left(\left(r_{1} / t\right) \cdot\left(\theta_{1}\right)\right)=3.532021 \cdot\left(\left(r_{1} / t\right) \cdot\left(\pi-\theta_{1}\right)\right)^{5.127981}-8.442514 \cdot\left(\left(r_{1} / t\right) \cdot\left(\pi-\theta_{1}\right)\right)^{3} \\
& +8.645182 \cdot\left(\left(r_{1} / t\right) \cdot\left(\pi-\theta_{1}\right)\right)^{2}+1.481668 \cdot\left(\left(r_{1} / t\right) \cdot\left(\pi-\theta_{1}\right)\right) \\
& +3.814145 \times 10^{-1}
\end{aligned}
$$




$$
\begin{aligned}
& f\left(L_{1} / t\right)=-1.140609 \times 10^{1} \cdot\left(L_{1} / t\right)^{3.036113}+7.436459 \times 10^{-2} \cdot\left(L_{1} / t\right)^{4}+1.188223 \times 10^{1} \cdot\left(L_{1} / t\right)^{3} \\
& -8.150334 \times 10^{-1} \cdot\left(L_{1} / t\right)^{2}+2.933742 \times 10^{-1} \cdot\left(L_{1} / t\right)+2.718308 \times 10^{-2} \\
& f\left(L_{2} / t\right)=1.919634 \times 10^{1} \cdot\left(L_{2} / t\right)^{-0.1508265}-7.315526 \times 10^{-1} \cdot\left(L_{2} / t\right)^{4}+4.967357 \cdot\left(L_{2} / t\right)^{3} \\
& -8.382442 \cdot\left(L_{2} / t\right)^{2}-5.482638 \cdot\left(L_{2} / t\right)+4.359819 \times 10^{1} \\
& f\left(\left(L_{1} / t\right) \cdot\left(L_{2} / t\right)\right)=-1.823407 \times 10^{-1} \cdot\left(\left(L_{1} / t\right) \cdot\left(L_{2} / t\right)\right)^{3}+1.469586 \cdot\left(\left(L_{1} / t\right) \cdot\left(L_{2} / t\right)\right)^{2} \\
& -5.862377 \cdot\left(\left(L_{1} / t\right) \cdot\left(L_{2} / t\right)\right)-8.301064 \\
& f(H / t)=-1.913752 \times 10^{1} \cdot(H / t)^{3}+7.690410 \cdot(H / t)^{2}-5.994878 \times 10^{-1} \cdot(H / t)+1.201826
\end{aligned}
$$

(3) Cruciform welded joint under tensile stress:

$$
\begin{aligned}
K_{t}= & 1+9.355385 \times 10^{-1} \cdot f(T / t) \cdot f\left(r_{1} / t\right) \cdot f\left(\theta_{1}\right) \cdot f\left(\left(r_{1} / t\right) \cdot\left(\theta_{1}\right)\right) \cdot f\left(L_{1} / t\right) \cdot f\left(L_{2} / t\right) . \\
& f\left(\left(L_{1} / t\right) \cdot\left(L_{2} / t\right)\right) \cdot f(H / t)
\end{aligned}
$$

where:

$f(T / t)=-4.241098 \times 10^{-1} \cdot(T / t)^{3}+1.392836 \cdot(T / t)^{2}-1.072866 \cdot(T / t)+5.223873$

$$
\begin{aligned}
f\left(r_{1} / t\right)= & -2.214616 \cdot\left(r_{1} / t\right)^{0.5081972}+3.365455 \cdot\left(r_{1} / t\right)^{3}-4.439046 \cdot\left(r_{1} / t\right)^{2}+3.726006 \cdot\left(r_{1} / t\right) \\
& +4.033244 \times 10^{-1}
\end{aligned}
$$$$
f\left(\theta_{1}\right)=-7.886656 \times 10^{-1} \cdot\left(\pi-\theta_{1}\right)^{3}+2.966534 \cdot\left(\pi-\theta_{1}\right)^{2}-5.878541 \cdot\left(\pi-\theta_{1}\right)+1.772818 \times 10^{1}
$$$$
f\left(\left(r_{1} / t\right) \cdot\left(\theta_{1}\right)\right)=1.557408 \cdot\left(\left(r_{1} / t\right) \cdot\left(\pi-\theta_{1}\right)\right)^{-0.1158765}+3.821716\left(\left(r_{1} / t\right) \cdot\left(\pi-\theta_{1}\right)\right)^{3}
$$

$$
\begin{aligned}
& -5.354226 \cdot\left(\left(r_{1} / t\right) \cdot\left(\pi-\theta_{1}\right)\right)^{2}+7.153383 \cdot\left(\left(r_{1} / t\right) \cdot\left(\pi-\theta_{1}\right)\right)-1.529427 \\
f\left(L_{1} / t\right)= & 8.158610 \cdot\left(L_{1} / t\right)^{1.944849}-1.90075 \times 10^{-2} \cdot\left(L_{1} / t\right)^{4}+2.021235 \times 10^{-1} \cdot\left(L_{1} / t\right)^{3}-7.977936 \cdot\left(L_{1} / t\right)^{2} \\
& -4.569099 \times 10^{-1} \cdot\left(L_{1} / t\right)+1.108427 \times 10^{-1}
\end{aligned}
$$

$$
\begin{aligned}
f\left(L_{2} / t\right)= & 1.020926 \times 10^{1} \cdot\left(L_{2} / t\right)^{0.09585178}+3.856400 \times 10^{-1} \cdot\left(L_{2} / t\right)^{4}-2.432551 \cdot\left(L_{2} / t\right)^{3} \\
& +6.069561 \cdot\left(L_{2} / t\right)^{2}-8.045796 \cdot\left(L_{2} / t\right)-5.364472
\end{aligned}
$$

$$
\begin{aligned}
f\left(\left(L_{1} / t\right) \cdot\left(L_{2} / t\right)\right)= & -2.421137 \times 10^{-1} \cdot\left(\left(L_{1} / t\right) \cdot\left(L_{2} / t\right)\right)^{3}+5.706142 \times 10^{-1} \cdot\left(\left(L_{1} / t\right) \cdot\left(L_{2} / t\right)\right)^{2} \\
& -2.159502 \times 10^{1} \cdot\left(\left(L_{1} / t\right) \cdot\left(L_{2} / t\right)\right)-2.240262
\end{aligned}
$$

$$
f(H / t)=4.996101 \cdot(H / t)^{3}-1.461031 \cdot(H / t)^{2}-1.874919 \times 10^{-1} \cdot(H / t)-1.083093
$$

(4) Cruciform welded joint under bending stress:

$$
\begin{gathered}
K_{t}=1+1.20077 \cdot f\left(r_{1} / t\right) \cdot f\left(\theta_{1}\right) \cdot f\left(\left(r_{1} / t\right) \cdot\left(\theta_{1}\right)\right) \cdot f\left(L_{1} / t\right) \cdot f\left(L_{2} / t\right) \cdot f\left(\left(L_{1} / t\right) \cdot\left(L_{2} / t\right)\right) \\
\text { where: } \\
\begin{array}{c}
f\left(r_{1} / t\right)=1.245919 \cdot\left(r_{1} / t\right)^{-0.1788861}+5.187295 \cdot\left(r_{1} / t\right)^{3}-5.888376 \cdot\left(r_{1} / t\right)^{2}+2.948041 \cdot\left(r_{1} / t\right)-2.024161 \\
f\left(\theta_{1}\right)=4.518553 \times 10^{-1} \cdot\left(\pi-\theta_{1}\right)^{3}-4.169720 \cdot\left(\pi-\theta_{1}\right)^{2}+6.471859 \cdot\left(\pi-\theta_{1}\right)+1.232356 \times 10^{1} \\
f\left(\left(r_{1} / t\right) \cdot\left(\theta_{1}\right)\right)=\quad 6.195167 \cdot\left(\left(r_{1} / t\right) \cdot\left(\pi-\theta_{1}\right)\right)^{1.958767}+2.102936 \times 10^{-1} \cdot\left(\left(r_{1} / t\right) \cdot\left(\pi-\theta_{1}\right)\right)^{3} \\
\quad-6.258362 \cdot\left(\left(r_{1} / t\right) \cdot\left(\pi-\theta_{1}\right)\right)^{2}+4.307112 \times 10^{-2} \cdot\left(\left(r_{1} / t\right) \cdot\left(\pi-\theta_{1}\right)\right)+1.578839 \times 10^{-2} \\
f\left(L_{1} / t\right)=1.069048 \times 10^{-1} \cdot\left(L_{1} / t\right)^{3}+4.896665 \times 10^{-1} \cdot\left(L_{1} / t\right)^{2}-3.181390 \cdot\left(L_{1} / t\right)+1.187674 \times 10^{1} \\
f\left(L_{2} / t\right)=\quad 6.217977 \cdot\left(L_{2} / t\right)^{2.938345}+8.220848 \times 10^{-2} \cdot\left(L_{2} / t\right)^{4}-5.872895 \cdot\left(L_{2} / t\right)^{3}-5.835022 \times 10^{-1} \cdot\left(L_{2} / t\right)^{2} \\
+1.809699 \times 10^{-1} \cdot\left(L_{2} / t\right)+2.220297 \times 10^{-2}
\end{array} \\
f\left(\left(L_{1} / t\right) \cdot\left(L_{2} / t\right)\right)=\quad-9.639892 \times 10^{-2} \cdot\left(\left(L_{1} / t\right) \cdot\left(L_{2} / t\right)\right)^{3}-2.213564 \times 10^{-1} \cdot\left(\left(L_{1} / t\right) \cdot\left(L_{2} / t\right)\right)^{2} \\
\quad+5.660853 \cdot\left(\left(L_{1} / t\right) \cdot\left(L_{2} / t\right)\right)+3.300823 \times 10^{1}
\end{gathered}
$$


The previously given text is shown below:

\section{Appendix B}

Formulas for calculating SCFs for T-shape welded joint and cruciform welded joint under tensile and bending stress.

(1) T-shape welded joint under tensile stress:

$$
K_{t}=1+1.394 \cdot f\left(r_{1} / t\right) \cdot f\left(\theta_{1}\right) \cdot f\left(\left(r_{1} / t\right) \cdot\left(\theta_{1}\right)\right)
$$

where:

$$
\begin{gathered}
f\left(r_{1} / t\right)=1.824 \cdot\left(r_{1} / t\right)^{-0.145}+6.400 \cdot\left(r_{1} / t\right)^{3}-6.802 \cdot\left(r_{1} / t\right)^{2}+3.277 \cdot\left(r_{1} / t\right)-2.692 \\
f\left(\theta_{1}\right)=-0.278 \cdot\left(\pi-\theta_{1}\right)^{3}+1.532 \cdot\left(\pi-\theta_{1}\right)^{2}-3.395 \cdot\left(\pi-\theta_{1}\right)+4.967 \\
f\left(\left(r_{1} / t\right) \cdot\left(\theta_{1}\right)\right)= \\
\begin{array}{c}
-10.016 \cdot\left(\left(r_{1} / t\right) \cdot\left(\pi-\theta_{1}\right)\right)^{3}+12.793 \cdot\left(\left(r_{1} / t\right) \cdot\left(\pi-\theta_{1}\right)\right)^{2}+ \\
5.761 \cdot\left(\left(r_{1} / t\right) \cdot\left(\pi-\theta_{1}\right)\right)+1.182
\end{array}
\end{gathered}
$$

(2) T-shape welded joint under bending stress:

$$
\begin{aligned}
& K_{t}=1-1.130 \cdot f(T / t) \cdot f\left(r_{1} / t\right) \cdot f\left(\theta_{1}\right) \cdot f\left(\left(r_{1} / t\right) \cdot\left(\theta_{1}\right)\right) \cdot f\left(L_{1} / t\right) \cdot f\left(L_{2} / t\right) \text {. } \\
& f\left(\left(L_{1} / t\right) \cdot\left(L_{2} / t\right)\right) \cdot f(H / t) \\
& \text { where: } \\
& f(T / t)=-0.010 \cdot(T / t)^{3}-0.032 \cdot(T / t)^{2}+0.185 \cdot(T / t)+2.433 \\
& f\left(r_{1} / t\right)=0.648 \cdot\left(r_{1} / t\right)^{-0.259}+2.861 \cdot\left(r_{1} / t\right)^{3}-3.690 \cdot\left(r_{1} / t\right)^{2}+2.090 \cdot\left(r_{1} / t\right)-1.239 \\
& f\left(\theta_{1}\right)=0.003 \cdot\left(\pi-\theta_{1}\right)^{3}-0.035 \cdot\left(\pi-\theta_{1}\right)^{2}+0.060 \cdot\left(\pi-\theta_{1}\right)+0.086 \\
& f\left(\left(r_{1} / t\right) \cdot\left(\theta_{1}\right)\right)=3.532 \cdot\left(\left(r_{1} / t\right) \cdot\left(\pi-\theta_{1}\right)\right)^{5.128}-8.443 \cdot\left(\left(r_{1} / t\right) \cdot\left(\pi-\theta_{1}\right)\right)^{3}+ \\
& 8.645 \cdot\left(\left(r_{1} / t\right) \cdot\left(\pi-\theta_{1}\right)\right)^{2}+1.482 \cdot\left(\left(r_{1} / t\right) \cdot\left(\pi-\theta_{1}\right)\right)+0.381 \\
& f\left(L_{1} / t\right)=-11.406 \cdot\left(L_{1} / t\right)^{3.036}+0.074 \cdot\left(L_{1} / t\right)^{4}+11.882 \cdot\left(L_{1} / t\right)^{3}-0.815 \cdot\left(L_{1} / t\right)^{2}+0.293 \cdot\left(L_{1} / t\right)+0.027 \\
& f\left(L_{2} / t\right)=19.196 \cdot\left(L_{2} / t\right)^{-0.151}-0.732 \cdot\left(L_{2} / t\right)^{4}+4.967 \cdot\left(L_{2} / t\right)^{3}-8.382 \cdot\left(L_{2} / t\right)^{2}-5.483 \cdot\left(L_{2} / t\right)+43.598 \\
& f\left(\left(L_{1} / t\right) \cdot\left(L_{2} / t\right)\right)=-0.182 \cdot\left(\left(L_{1} / t\right) \cdot\left(L_{2} / t\right)\right)^{3}+1.470 \cdot\left(\left(L_{1} / t\right) \cdot\left(L_{2} / t\right)\right)^{2}-5.862 \cdot\left(\left(L_{1} / t\right) \cdot\left(L_{2} / t\right)\right)-8.301 \\
& f(H / t)=-19.138 \cdot(H / t)^{3}+7.690 \cdot(H / t)^{2}-0.599 \cdot(H / t)+1.202 \\
& \text { (3) Cruciform welded joint under tensile stress: } \\
& K_{t}=1+0.936 \cdot f(T / t) \cdot f\left(r_{1} / t\right) \cdot f\left(\theta_{1}\right) \cdot f\left(\left(r_{1} / t\right) \cdot\left(\theta_{1}\right)\right) \cdot f\left(L_{1} / t\right) \cdot f\left(L_{2} / t\right) \cdot \\
& f\left(\left(L_{1} / t\right) \cdot\left(L_{2} / t\right)\right) \cdot f(H / t) \\
& \text { where: } \\
& f(T / t)=-0.424 \cdot(T / t)^{3}+1.393 \cdot(T / t)^{2}-1.073 \cdot(T / t)+5.224 \\
& f\left(r_{1} / t\right)=-2.215 \cdot\left(r_{1} / t\right)^{0.508}+3.365 \cdot\left(r_{1} / t\right)^{3}-4.439 \cdot\left(r_{1} / t\right)^{2}+3.726 \cdot\left(r_{1} / t\right)+0.403 \\
& f\left(\theta_{1}\right)=-0.789 \cdot\left(\pi-\theta_{1}\right)^{3}+2.967 \cdot\left(\pi-\theta_{1}\right)^{2}-5.879 \cdot\left(\pi-\theta_{1}\right)+17.728 \\
& f\left(\left(r_{1} / t\right) \cdot\left(\theta_{1}\right)\right)=1.557 \cdot\left(\left(r_{1} / t\right) \cdot\left(\pi-\theta_{1}\right)\right)^{-0.116}+3.822 \cdot\left(\left(r_{1} / t\right) \cdot\left(\pi-\theta_{1}\right)\right)^{3}-5.354 \cdot\left(\left(r_{1} / t\right) \cdot\left(\pi-\theta_{1}\right)\right)^{2}+ \\
& 7.153 \cdot\left(\left(r_{1} / t\right) \cdot\left(\pi-\theta_{1}\right)\right)-1.529 \\
& f\left(L_{1} / t\right)=8.159 \cdot\left(L_{1} / t\right)^{1.945}-0.019 \cdot\left(L_{1} / t\right)^{4}+0.202 \cdot\left(L_{1} / t\right)^{3}-7.978 \cdot\left(L_{1} / t\right)^{2}-0.457 \cdot\left(L_{1} / t\right)+0.111 \\
& f\left(L_{2} / t\right)=10.209 \cdot\left(L_{2} / t\right)^{0.096}+0.386 \cdot\left(L_{2} / t\right)^{4}-2.433 \cdot\left(L_{2} / t\right)^{3}+6.070 \cdot\left(L_{2} / t\right)^{2}-8.046 \cdot\left(L_{2} / t\right)-5.364 \\
& f(H / t)=4.996 \cdot(H / t)^{3}-1.461 \cdot(H / t)^{2}-0.187 \cdot(H / t)-1.083 \\
& f\left(\left(L_{1} / t\right) \cdot\left(L_{2} / t\right)\right)=-0.242 \cdot\left(\left(L_{1} / t\right) \cdot\left(L_{2} / t\right)\right)^{3}+0.571 \cdot\left(\left(L_{1} / t\right) \cdot\left(L_{2} / t\right)\right)^{2}-21.595 \cdot\left(\left(L_{1} / t\right) \cdot\left(L_{2} / t\right)\right)-2.240
\end{aligned}
$$


(4) Cruciform welded joint under bending stress:

$$
\begin{aligned}
& K_{t}=1+1.201 \cdot f\left(r_{1} / t\right) \cdot f\left(\theta_{1}\right) \cdot f\left(\left(r_{1} / t\right) \cdot\left(\theta_{1}\right)\right) \cdot f\left(L_{1} / t\right) \cdot f\left(L_{2} / t\right) \text {. } \\
& f\left(\left(L_{1} / t\right) \cdot\left(L_{2} / t\right)\right) \\
& \text { where: } \\
& f\left(r_{1} / t\right)=1.246 \cdot\left(r_{1} / t\right)^{-0.179}+5.187 \cdot\left(r_{1} / t\right)^{3}-5.888 \cdot\left(r_{1} / t\right)^{2}+2.948 \cdot\left(r_{1} / t\right)-2.024 \\
& f\left(\theta_{1}\right)=0.452 \cdot\left(\pi-\theta_{1}\right)^{3}-4.170 \cdot\left(\pi-\theta_{1}\right)^{2}+6.472 \cdot\left(\pi-\theta_{1}\right)+12.324 \\
& f\left(\left(r_{1} / t\right) \cdot\left(\theta_{1}\right)\right)=6.195 \cdot\left(\left(r_{1} / t\right) \cdot\left(\pi-\theta_{1}\right)\right)^{1.959}+0.210 \cdot\left(\left(r_{1} / t\right) \cdot\left(\pi-\theta_{1}\right)\right)^{3}-6.258 \cdot\left(\left(r_{1} / t\right) \cdot\left(\pi-\theta_{1}\right)\right)^{2}+ \\
& 0.043 \cdot\left(\left(r_{1} / t\right) \cdot\left(\pi-\theta_{1}\right)\right)+0.016 \\
& f\left(L_{1} / t\right)=0.107 \cdot\left(L_{1} / t\right)^{3}+0.490 \cdot\left(L_{1} / t\right)^{2}-3.181 \cdot\left(L_{1} / t\right)+11.877 \\
& f\left(L_{2} / t\right)=6.218 \cdot\left(L_{2} / t\right)^{2.938}+0.082 \cdot\left(L_{2} / t\right)^{4}-5.873 \cdot\left(L_{2} / t\right)^{3}-0.584 \cdot\left(L_{2} / t\right)^{2}+0.181 \cdot\left(L_{2} / t\right)+0.022 \\
& f\left(\left(L_{1} / t\right) \cdot\left(L_{2} / t\right)\right)=-0.096 \cdot\left(\left(L_{1} / t\right) \cdot\left(L_{2} / t\right)\right)^{3}-0.221 \cdot\left(\left(L_{1} / t\right) \cdot\left(L_{2} / t\right)\right)^{2}+5.661 \cdot\left(\left(L_{1} / t\right) \cdot\left(L_{2} / t\right)\right)+33.008
\end{aligned}
$$

The authors would like to apologize for any inconvenience caused to the readers by these changes.

\section{Reference}

1. Wang, Y.; Luo, Y.; Tsutsumi, S. Parametric Formula for Stress Concentration Factor of Fillet Weld Joints with Spline Bead Profile. Materials 2020, 13, 4639. [CrossRef] 